\title{
reviews
}

\section{Physiology of Octopus}

J. B. Messenger

Octopus: Physiology and Behaviour of an Advanced Invertebrate. By M. J. Wells. Pp. 417. (Chapman and Hall: London, 1977.) £17.

LoNG before J. Z. Young and his colleagues began the detailed investigations of the nervous system that have recently made the octopus so famous, physiologists had studied its heart rate and colour change mechanism, its blood and its reproductive system. There is an extensive recent literature, too, on many aspects of non-nervous physiology and $\mathrm{Dr}$ Wells has attempted to review all this and set it in the context of the behaviour of the animal. He has also re-examined the now considerable body of work on the brain and sense organs.

Although he states that his aim has been "to produce a comprehensive physiology of a single invertebrate type" there are bound to be doubts about how far he has achieved this. And these doubts will not be allayed by his cavalier confession that: "I have never, personally, been able to find much excitement in chemistry and I have cravenly evaded discussion at levels where I am frankly incompetent and reluctant to do the necessary homework". (Ammunition for tutorial students here.) "A monograph . . . is bound to be skewed by too much print squandered on things that interest the author, and too little on the things that may interest others. I am sorry about this. But not very". It should be said at once, however, that this book will be extremely useful for an undergraduate, for a postgraduate beginning his research into cephalopods, or for anyone anxious to find his way into the literature; the work is well documented and there is an extensive reference list. Whether such people will themselves lay out $£ 17$ is something that probably only the publishers need worry about. But surely the price could have been kept down-for example, by omitting the colour plates and even the half-tones?

The first two-fifths of the book deal with the non-nervous physiology. After an introduction touching on the history of cephalopods, comes an "outline of the anatomy"; experts will regret that there is nothing new here (the diagram of the brain is a familiar one from Young and the three figures of the circulatory system are Isgrove's 70-yr-old drawings of Eledone) but someone who has never seen or dissected an octopus will certainly find it helpful. Then there follows a series of very interesting chapters on respiration, circulation, excretion, digestion, reproduction and endocrinology. There is a wealth of information here, nearly all brought together for the first time, much of it in the form of line diagrams, and well-chosen graphs and tables (often most usefully combining data from more than one author). Chapter 3 includes unpublished results from Dr Wells' own experiments, confirming that the heartbeat of Octopus is very slow to regain its 'resting' level after quite moderate exercise. Equally interesting is his discovery that if octopuses experience sexual 'excitement' it is not manifest at the level of the circulation: no changes in heartbeat frequency or amplitude are detectable in males during copulation. These are only two of several examples that the author uses to point up his recurrent theme that octopuses are phylogenetically remote from ourselves, so that they may be expected to show features that reflect their molluscan origins rather than their vertebrate habits. Obvious to a zoologist, perhaps, but a valuable reminder to a mammalian physiologist or a psychologist, that the perceptual world and motivating systems of octopuses must be very different from our own.

In the middle fifth, which deals with the sense organs and sensory processing, this idea is pursued as the author reviews his own work on the curiously (by vertebrate standards) restricted use of proprioceptive information by Octopus. Vision is dealt with very fully in this section but a number of interesting points are left in the air. For example, is there a 'fovea' in the centre of the retina, as Tasaki's work and the experiments of Muntz imply? How do we reconcile behavioural evidence that Octopus can discriminate between two sources of polarised light when the $e$ vectors lie at $45^{\circ}$ and $135^{\circ}$ with electrophysiological findings that this is impossible at the retinal level? And is the cephalopod retina really so "simple"? The recent experiments of Lange and Hartline suggest that functionally it may be quite complex. Of course, these are detailed criticisms but it seems important in a book like this to draw attention to areas of ignorance - indeed, Dr Wells is very clear about this later on when dealing with learning and the nervous system.

The final two-fifths of the book are devoted to these topics and also includes a chapter on motor control. It is difficult for someone working in this field to see the wood for the trees here. Undoubtedly we must all be grateful to Dr Wells for summarising so much information, but could it not have been done with a bolder touch? In the chapter on tactile learning, for example, there is a wealth of detail that makes parts of it almost totally opaque. Happily, this is not true of the last chapter, on visual learning, which ends on a stimulating note with an account of the author's model of an associative learning system based on the connectivity of the vertical lobe.

On the whole, the balance of the book seems just right, given that we know so much more about the brain and sense organs. It is the omissions that will cause eyebrows to be raised. For example, there is no mention of jet propulsion, a most characteristic feature of this and other cephalopods. In a lengthy section on the optic gland, an alternative hypothesis that has recently been suggested for its function is completely ignored. And, most extraordinary of all, nothing is said about the physiology of nerves. The index does not include "action potential", "axoplasm", "sodium pump" or "synapse". Of course, virtually all the now classic work on cephalopod neurophysiology has been carried out in squids but these are closely related to octopuses and the work is of such coherence and importance that it seems strange to omit it altogether.

Perhaps Dr Wells was reluctant to do his homework here, but what is really called into question is the wisdom of writing a book on one species when its biology has not been comprehensively studied. A Physiology of Rhodnius might seem a fine idea but I suspect its author would hesitate when he came to the sense organs. What should he do then: omit the entire work of the Canberra and Tübingen schools or write a Physiology of insects? A daunting task indeed; but a Physiology of Cephalopods need only draw on data from two other genera (Sepia and Loligo) to be complete! In fact this is not a Physiology of Octopus at all but an account of areas of Octopus biology that interest the author. The result is very readable, well illustrated and likely to be a source book for some time. It is not, however, an authoritative or definitive work such as we would expect of someone like Dr Wells who has himself made important contributions to the physiology of Octopus. I am sorry to have to say this. But not very.

J. B. Messenger is Senior Lecturer in Zoology at the University of Sheffield, UK. 